\section{Elektrische Entladungen in Strahlen aus kondensiertem Wasserstoff und kondensiertem Stickstoff im Hochvakuum}

\author{
Von E. W. Becker und R. Klingelhöfer
}

Kernforschungszentrum Karlsruhe, Institut für Kernverfahrenstechnik der Technischen Hochschule

(Z. Naturforschg. 19 a, 813-814 [1964] ; eingegangen am 22. Mai 1964)

Vor einiger Zeit haben wir vorgeschlagen, Strahlen aus sehr kalten, durch vaN-DER-W $-W_{\text {AALS}}$-Kräfte zusammenhängenden Deuterium- bzw. Deuterium-TritiumAgglomeraten ${ }^{1}$ zur gerichteten Einführung des Kernbrennstoffes in den hoch evakuierten Reaktionsraum von Kernfusionsanlagen zu verwenden ${ }^{2,3}$. Ein spezieller Vorschlag ging davon aus, daß sich in solchen Strahlen aus kondensierter Materie im Hochvakuum elektrische Entladungen zünden lassen, die die Molekül-Agglomerate genügend schnell in ein räumich scharf begrenztes Plasma umwandeln. Die Zündung derartiger Entladungen ist in der Zwischenzeit gelungen, worüber im folgenden kurz berichtet werden soll:

Der Strahl wurde unter Verwendung eines früher beschriebenen Kryostaten ${ }^{4}$ mit einer durch flüssigen Wasserstoff gekühlten LavaL-Düse erzeugt, die mit einem elektromagnetisch betätigten Schnellschlußventil ${ }^{\mathbf{5}}$ versehen war. Zur Auslösung der Entladung diente die in Abb. 1 dargestellte Anordnung, die folgendermaßen arbeitet: Der Strahl wird bei aufgeladenem

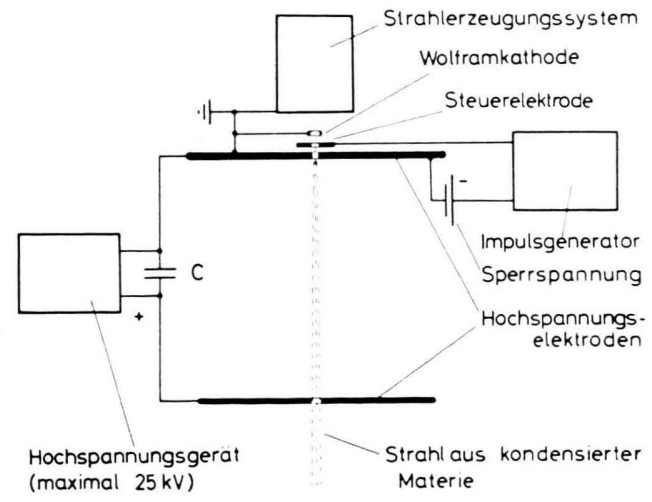

Abb. 1. Anordnung zur Erzeugung elektrischer Entladungen in Strahlen aus kondensiertem Wasserstoff bzw. kondensiertem Stickstoff im Hochvakuum.

Kondensator C für kurze Zeit in den hoch evakuierten Raum zwischen den Hochspannungselektroden eingeschossen. Durch den Spulenstrom des Schnellschlußventils wird mit einstellbarer Verzögerung der ImpulsGenerator ausgelöst, der die Steuerelektrode vorüber-

1 E. W. Becker, K. Bier u. W. Henkes, Z. Phys. 146, 33 [1956].

2 E. W. Becker, Beams of Condensed Matter in High Vacuum, Vortrag auf der Brookhaven Conference on Molecular Beams, Heidelberg, 11. 6. 1959. gehend auf positives Potential gegenüber der elektrisch geheizten, ringförmigen Wolframkathode bringt. Die in den Strahl eintretenden Elktronen zünden die Entladung zwischen den Hochspannungselektroden längs des Strahls.

Die Versuche wurden mit einem Gemisch aus $50 \mathrm{Mol}$ Proz. $\mathrm{H}_{2}$ und 50 Mol-Proz. He durchgeführt. Der Heliumzusatz, der vor dem Eintritt des Strahls in den hoch evakuierten Entladungsraum praktisch vollständig aus dem Strahl ausscheidet, bewirkt eine erhebliche Vergrößerung der im Strahl erreichbaren Wasserstoffdichte ${ }^{4}$.

Bei einem typischen Experiment betrug der Einlaßdruck des Gasgemisches 7,0 ata, der Druck über dem Wasserstoffbad 2,4 ata und die Öffnungszeit des Düsenventils ca. $1 \cdot 10^{-2}$ sec. Die Laval-Düse hatte einen engsten Durchmesser von $0,9 \mathrm{~mm}$. Die Wasserstoffmoleküldichte betrug in der Mitte zwischen den $22 \mathrm{~cm}$ voneinander entfernten Hochspannungselektroden etwa $10^{16}$ $\mathrm{H}_{2}$-Moleküle $/ \mathrm{cm}^{3}$ bei einem Strahldurchmesser an dieser Stelle von ca. $4 \mathrm{~mm}$. Der Umgebungsdruck lag unter $2 \cdot 10^{-6} \mathrm{~mm} \mathrm{Ho}$.

Abb. 2 a zeigt die mit einer normalen Kamera ohne Zeitauflösung photographierte Leuchterscheinung, wie sie sich unter diesen Bedingungen mit einem $0,1 \mu \mathrm{F}$. Kondensator und einer Ladespannung von $15 \mathrm{kV}$ er-

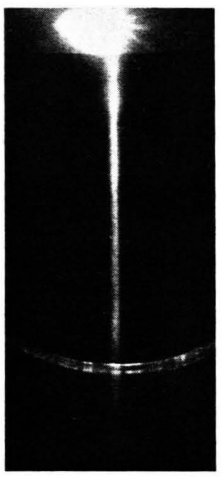

2 a.

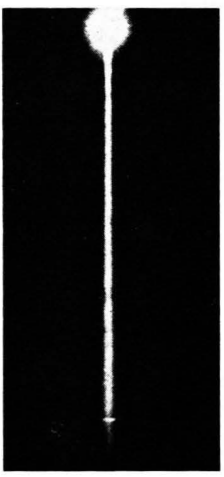

2 b.
Abb. 2. Mit einer normalen Kamera ohne Zeitauflösung photographierte elektrische Entladungen in Strahlen aus kondensiertem Wasserstoff (a) und kondensiertem Stickstoff (b) im Hochvakuum.

gibt. Der Strahl läuft in dem Bild von oben nach unten. Die horizontale, leicht gekrümmte Leuchterscheinung im untersten Drittel des Bildes ist ein Lichtreflex an dem Rande des Pumpstutzens. Die Periode des Entladungsstromes betrug ungefähr $8 \cdot 10^{-7} \mathrm{sec}$, die Dämpfungskonstante ca. $2 \cdot 10^{-6}$ sec. Aus der Periode, der Kapazität und der Ladespannung errechnet man eine Stromspitze von ca. $7 \mathrm{kAmp}$.

3 E. W. Becker, R. Klingelhöfer u. P. Lohse, Z. Naturforschg. 15 a, 644 [1960].

4 E. W. Becker, R. Klingelhöfer u. P. Lohse, Z. Naturforschg. 17 a, 432 [1962].

5 O. Hagena, Z. Angew. Phys. 16, 183 [1963]. 
Abb. 2 b zeigt das Bild einer mit einem 10-mal kleineren Kondensator bei derselben Spannung in einem Strahl aus kondensiertem Stickstoff erzeugten Entladung. Die Periode war hier ungefähr $5 \cdot 10^{-7}$ sec. Die Leuchterscheinung ist räumlich noch schärfer begrenzt als in Abb. 2 a. Auch beim Wasserstoff ergeben sich mit dem kleineren Kondensator räumlich schärfer begrenzte Leuchterscheinungen. Sie lieferten jedoch mit der ver-

${ }^{6}$ J. G. Linhart, Nuovo Cim. 17, 850 [1960]. - Ch. Maisonnier, J. G. Linahrt u. M. Haegi, Nuclear Fusion, Suppl. 2, 727 [1962].

\section{Protonenrelaxation und Hydratation in wäßrigen Lösungen diamagnetischer Salze}

\author{
Von W. Lohmann
}

Physikalisches Institut der Karl-Marx-Universität, Leipzig (Z. Naturforschg. 19 a, $814-816$ [1964] ; eingegangen am 8. Mai 1964)

Messungen der Protonenrelaxationszeiten von wäßrigen Lösungen diamagnetischer Salze wurden bisher nur bezüglich der longitudinalen Relaxationszeit $T_{1}$ veröffentlicht ${ }^{1-3}$. Bei den Salzen, die als strukturbildend bekannt sind, sind die Relaxationszeiten der Lösung verkürzt gegenüber denen des reinen Wassers. Der Einfluß auf die Relaxationsraten hat zwei Ursachen:

I. Die Bewegung des Ionenkomplexes ist langsamer als die der freien Wassermoleküle, d. h. die Korrelationszeit $\tau_{\mathrm{c}}$ der in der ersten Koordinationssphäre haftenden Wassermoleküle ist größer als die Korrelationszeit $\tau_{\text {c } 0}$ der freien Wassermoleküle.

II. Es findet chemischer Austausch der Protonen zwischen Bereichen mit verschiedenem mittleren $H_{0}$-Feld statt, und zwar dem Bereich a des freien Wassers und dem Bereich b der Hydrathülle ${ }^{4}$ des Ions. Der Einfluß I erstreckt sich auf die longitudinale Relaxationszeit $T_{1}$ und die transversale Relaxationszeit $T_{2}$ in gleicher Weise, der chemische Austausch hingegen beeinflußt nur $T_{2}$. Dies hat zur Folge, daß die transversale Relaxationszeit der Lösung gegenüber der des freien Wassers stärker verkürzt sein sollte als die longitudinale Relaxationszeit.

Bei schnellem Austausch der Protonen zwischen der Hydrathülle und dem Bereich des übrigen Lösungswassers ergibt der Vorgang I eine resultierende Relaxationsrate, die dem gewichteten Mittel der Relaxationsraten $1 / T_{10}$ der Protonen im Bereich a und $1 / T_{1 \mathrm{H}}$ der der Hydrathülle entspricht. Bei reinem Wasser gilt ${ }^{5}$

$$
\begin{aligned}
\frac{1}{T_{10}} & =\frac{3 \gamma^{4} \hbar^{2}}{2 b^{6}} \tau_{\mathrm{c} 0}+\frac{2 \pi}{5} \frac{N \gamma^{4} \hbar^{2}}{a D} K \\
& =\left(1 / T_{10}\right)_{\operatorname{rot}}+\left(1 / T_{10}\right)_{\text {trans }} .
\end{aligned}
$$

1 S. Broersma, J. Chem. Phys. 24, 659 [1956]; 27, 481 [1957].

2 W. M. W $1,28,122,268$ [1960].

${ }^{3}$ H. G. Hertz, Ber. Bunsenges. phys. Chem. 67, 311 [1963]. wendeten Kamera keine für die Wiedergabe im Druck geeigneten Bilder.

Es soll versucht werden, nach der beschriebenen Methode einen dünnwandigen Plasmahohlzylinder aus Deuterium bzw. Deuterium-Tritium-Gemischen im Hochvakuum aufzubauen. Dünnwandige Plasmahohlzylinder mit kleinem Verhältnis von Länge zu Durchmesser sind für die von LinHaRT ${ }^{6}$ vorgeschlagenen schnellen z-pinchEntladungen von Interesse. $\mathrm{Da}$ sich nach unserer Methode voraussichtlich auch dünnwandige Plasmahohlzylinder mit großem Verhältnis von Länge zu Durchmesser erzeugen lassen, erscheint auch eine Kompression der Plasmaschicht mit einem $\Theta$-Feld aussichtsreich.

Der erste Term berücksichtigt den Einfluß der rotatorischen Diffusion und der zweite Term den der translatorischen Diffusion. Es ist

$\gamma \quad$ das gyromagnetische Verhältnis des Protons.

$b$ der Abstand der Protonen im Wassermolekül,

$N$ die Anzahl der Wassermoleküle pro Volumen,

$a$ der minimale Abstand zweier Protonen, die nicht zum gleichen Wassermolekül gehören,

$D$ der Diffusionskoeffizient der Translation,

$K$ ein Korrekturfaktor, der von der Struktur der Flüssigkeit abhängt.

Bezüglich der Größe von $K$ läßt sich folgendes aussagen: Im Fall einer idealen Flüssigkeit wäre $K=1$; im Spezialfall des flächenzentrierten kubischen Gitters wird ${ }^{6} K=75 / 40$. Man darf annehmen, daß im Wasser der Wert von $K$ zwischen 1 und 2 liegen wird; die genaue Größe von $K$ ist aber für die weiteren Betrachtungen von untergeordneter Bedeutung. - Setzt man in Gl. (1) die entsprechenden Zahlenwerte ein, und vergleicht man mit dem experimentellen Wert für $T_{10}$, so findet man, daß $\left(1 / T_{10}\right)_{\text {trans }} \approx\left(1 / T_{10}\right)_{\text {rot }}$ ist.

Für die Relaxationsrate $1 / T_{1}$ der Elektrolytlösung erhält man

$$
\begin{gathered}
\frac{1}{T_{1}}=p_{\mathrm{a}} \frac{3 \gamma^{4} \hbar^{2}}{2 b^{6}} \tau_{\mathrm{c} 0}+p_{\mathrm{a}} \frac{2 \pi}{5} \frac{N \gamma^{4} \hbar^{2}}{a D} K \\
\quad+p_{\mathrm{l}} \frac{3 \gamma^{4} \hbar^{2}}{2 b^{6}} \tau_{\mathrm{c}}+p_{\mathrm{b}} \frac{2 \pi}{5} \frac{N \gamma^{4} \hbar^{2}}{a^{\prime} D^{\prime}} f K^{\prime} \\
\text { mit } p_{\mathrm{a} \mathrm{a}}=1-\frac{m N_{\mathrm{I}}}{N} \text { und } p_{\mathrm{b}}=\frac{m N_{\mathrm{I}}}{N} .
\end{gathered}
$$

Hierbei sind $a^{\prime}, D^{\prime}$ und $K^{\prime}$ die Größen für die Wassermoleküle im Hydratkomplex analog zu $a, D$ und $K$ im freien Wasser; auf den Faktor $f$ wird weiter unten eingegangen. Die Molfraktionen $p_{\mathrm{a}}$ und $p_{\mathrm{b}}$ der Protonen in den Bereichen a bzw. b erhält man aus der Konzentration der Kationen $\left(N_{\mathrm{I}}\right.$ ist die Anzahl der Kationen pro Volumen) und der primären Hydratationszahl $m$. Weshalb der Einfluß der Anionen hier vernachlässigt werden kann, wird weiter unten begründet.

${ }^{4}$ Hydrathülle sei die Kurzbezeichnung für die Wassermoleküle der ersten Koordinationssphäre.

5 A. Abragam, The Principles of Nuclear Magnetism, Oxford University Press 1961

${ }^{6}$ Siehe $^{5}$, S. 462. 